\title{
Gadolinium-mesoporous Silica as a Potential Magnetic Resonance Imaging Contrast Agent
}

\section{DIANA RAKHMAWATY EDDY ${ }^{1 *}$, ANNI ANGGRAENI ${ }^{1}$, RETNA PUTRI FAUZIA ${ }^{1}$, IMAN RAHAYU', ABDUL MUTALIB ${ }^{1}$, M. LUTFI FIRDAUS ${ }^{2}$ and HUSEIN HERNANDI BAHTI ${ }^{1}$}

\author{
1'Department of Chemistry, Universitas Padjadjaran, Bandung, Indonesia. \\ ${ }^{2}$ Graduate School of Science Education, University of Bengkulu, Bengkulu, Indonesia. \\ *Corresponding author E-mail: diana.rahmawati@unpad.ac.id
}

http://dx.doi.org/10.13005/ojc/340550

(Received: July 27, 2018; Accepted: August 29, 2018)

\begin{abstract}
Gadolinium-mesoporous silica (Gd-MS) has been used widely as an alternative for synthesizing gadolinium-based Magnetic Resonance Imaging (MRI) contrast agents. In this study, Gd-MS was prepared by using the sol-gel method. Transmission Electron Microscopy (TEM) results gave an average pore size value of $38.184 \mathrm{~nm}$, while the surface value of gadolinium mesoporous silica which is high at $580,666 \mathrm{~nm}$. We describe the synthesis, characterization, and stability test of a novel type of magnetic nanoparticles based on Gd-Si oxide. The particles are nanosized and can enter cell easily. This is a completely novel biomedical application of mesoporous silica materials.
\end{abstract}

Keywords: Gadolinium, Silica, Mesoporous, Contrast agents, Sol-gel.

\section{INTRODUCTION}

In recent years, magnetic resonance imaging (MRI) has emerged as one of the best techniques in medical diagnosis. MRI has been widely used and promotes the development of pharmaceutical compounds called contrast agents to provide contrast between normal tissue and diseased tissue and to show the status of organ function or blood flow. Non-invasive (selective) MRI detects morphological, functional, and most sensitive changes to soft tissue ${ }^{1}$.

Commercial contrast agents are dominated by $\mathrm{Gd}$ complexes involving multidentate ligands for high stability such as Magnevist [Gd (DTPA) $\left.\left(\mathrm{H}_{2} \mathrm{O}\right)\right]^{2-}$, DTPA is diethylenetri-aminepentaacetic acid and dotarem [Gd (DOTA) $\left(\mathrm{H}_{2} \mathrm{O}\right)$ ], DOTA is $1,4,7$, 10-tetraazacyclododecane-1,4,7,10-tetraacetic acid, each of which is covalently joined to the surface of the particle. The particles are modified to be characterized as gadolinium content containing proton relaxivity, which is a factor in determining contrast in MRI ${ }^{2}$.

The recent evolution of molecular imaging has resulted in further demand for more sensitive contrast compounds. Gadolinium in complex form

This is an Open Access article licensed under a Creative Commons Attribution-Non Commercial-Share Alike 4.0 International License (https://creativecommons.org/licenses/by-nc-sa/4.0/), which permits unrestricted Non Commercial use, distribution and reproduction in any medium, provided the original work is properly cited. 
has a strong impact on relaxation proton water because it contains seven unpaired electrons and slow electron spin relaxation from gadolinium (III). Gd (III) is freely toxic to the human body, hence chelating is used to form stable and inert complexes, which can be applied as contrast agents ${ }^{3}$.

Gadolinium with multidentate ligands such as DTPA have high complex stability but provide some side effects such as headache, nausea, and burning sensation at the injection site $^{1}$, whereas the use of silica nanoparticles as enzyme carriers can overcome many limitations, because silica is a powerful, non-toxic, ultrasound-sized particle that can be easily removed from the body through the kidneys. Silica nanoparticles are useful in many applications, such as biosensors, in vivo tracers, biomarkers of dye nanoparticulate markers, and delivery of drugs and genes due to their high biocompatibility and low toxicity ${ }^{4}$.

Research on nanoscale silica material has been highly developed, especially in mesoporous size because of its superiority in various applications. The mesoporous material is more directed in the formation of a regular mesostructure. Selection of basic materials mesoporous material is important because it acts as a building block. One of the underlying ingredients is silica because it has thermally stable and harmless properties ${ }^{5}$.

The gadolinium contrast agent is a chemical compound used in MRI scans. When injected into the body, gadolinium contrast agents will increase its relaxivity, which will improve the quality of MRI images. This allows radiologists to report more accurately how the body works and whether there are diseases or abnormalities. The gadolinium contrast agent consists of complex molecules and the arrangement of atoms united by chemical bonds. A chemical bond is made between the gadolinium ion and the carrier molecule (chelate). The carrier agent prevents the toxic nature of gadolinium by maintaining its contrasting properties. Contrast agents are injected intravenously (to the blood vessels) as part of an MRI scan which is then eliminated from the body via the kidneys ${ }^{6}$.

Therefore, research on a method for incorporating gadolinium into the mesoporous silica framework is based on its potential as an
MRI contrast agent because silica has a thermally stable, harmless, and easily available property ${ }^{5}$. The interaction between metal and silica tissue makes it less susceptible to leaching, in contrast to the ion-exchange metal species ${ }^{7}$.

\section{MATERIALS AND METHODS}

\section{Materials}

The chemicals used in the study were ammonia $25 \%$, glacial acetic acid, chloride acid, cetyltrimethylammonium bromide (CTAB), gadolinium (III) oxide $\left(\mathrm{Gd}_{2} \mathrm{O}_{3}\right)$, demineralized water, sodium acetate buffer solution, sodium-ethylene diamine tetraacetate (Na-EDTA), sodium hydroxide, tetraethyl orthosilicate (TEOS), and xylenol orange.

\section{Methods \\ Synthesis of Mesoporous Gd-Silica}

A total of $1.60 \mathrm{mmol}$ cetyltrimethylammonium bromide (CTAB) was dissolved in $29 \mathrm{~g}$ of distilled water and then $3.3 \mathrm{~mL}$ of $37 \% \mathrm{HCl}$ was added to the stirred solution strongly at $32^{\circ} \mathrm{C}$. Then $7.2 \mathrm{mmol}$ of tetraethyl orthosilicate (TEOS) was added to the water-soluble CTAB solution and addition of $\mathrm{HCl}$.

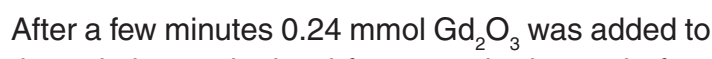
the solution and stirred for several minutes before pouring a $0.5 \mathrm{M}$ ammonium solution with final $\mathrm{pH}$ being achieved to 9 . The fine particle sludge was produced and dried at $80^{\circ} \mathrm{C}$, then calcined at $580^{\circ}$ $\mathrm{C}$ for $6 \mathrm{~h}$ to remove the template ${ }^{8}$.

The determination of gadolinium (III) is free with visible light spectrophotometry.

Determination of maximum wavelength of xylenol orange.

An aqueous xylenol solution of $12.80 \mathrm{ppm}$ in an acetate buffer solution was measured using a visible light spectrophotometer at a wavelength of $400 \mathrm{~nm}$ to $700 \mathrm{~nm}$. The wavelength indicating the maximum absorption was recorded as the maximum absorption wavelength of the xylenol orange ${ }^{9}$.

\section{The free gadolinium (III) calibration curves}

Calibration curve of free Gd (III) was made with four variations of concentration. The standard solution $\mathrm{Gd}$ (III) 100.00 ppm of $\mathrm{Gd}_{2} \mathrm{O}_{3}$ dissolved using nitric acid was diluted into four standard solutions Gd (III) with a concentration of 2.00; 3.00; 4.00; and 
$5.00 \mathrm{ppm}$. Each of them is $0.2 ; 0.3 ; 0.4$; and $0.5 \mathrm{~mL}$ Gd (III) 100.00 ppm were then added each of 2, 3, 4 and $5 \mathrm{~mL}$ of xylenol orange $40.00 \mathrm{ppm}$ (Gd (III): xylenol orange $=1: 10)^{1}$.

The standard solution which has been prepared is then measured uptake at the maximum wavelength of xylenol orange with visible light spectrophotometer. Furthermore, the calibration curve of Gd (III) is made from the data obtained to obtain the equation of the line ${ }^{1}$.

\section{Determination of Gd (III) on Gd-MS synthesis}

Gd-MS synthesis was suspended with distilled water, and then the filtrate was taken as much as $0.3 \mathrm{~mL}$ and dissolved with a sodium acetate buffer solution of $\mathrm{pH} 5.8$ to $3 \mathrm{~mL}$ and then added xylenol orange $40 \mathrm{ppm}$ (1:10).

The solution was added with a sodium acetate buffer solution of $\mathrm{pH} 5.8$ to the mark in the measuring flask. Then the solution was measured uptake at the maximum wavelength of xylenol orange ${ }^{1}$.

\section{Gd-MS stability test}

The stability was tested by immersing Gd-MS in a solution of distilled water $\mathrm{pH} 6$ at $37.5^{\circ} \mathrm{C}$ for 24 hours. After centrifugation, the supernatant was adjusted to the addition of hydrochloric acid to $\mathrm{pH} 4$ and added sodium buffer solution acetate, then titrated with EDTA $0.02 \mathrm{~N}$ using xylenol orange as an indicator ${ }^{8}$.

\section{RESULTS AND DISCUSSION}

\section{Synthesis of Mesoporous Silica Gadolinium Powder}

The synthesis of $\mathrm{Gd}-\mathrm{MS}$ involves several components, which are gadolinium (III) oxide, tetraethylorthosilicate (TEOS), cetyltrimethylammonium bromide (CTAB), and hydrochloric acid as catalysts. Gadolinium (III) oxide was used as a gadolinium source which is the main subject as a contrast agent. Gadolinium (III) oxide is insoluble in water, so it requires hydrochloric acid as a gadolinium solvent in the system and also acts as a catalyst that can accelerate the synthesis process. TEOS is a component that becomes the source of silica that will act as a carrier of gadolinium in body tissues. Precursors commonly used in the study of the formation of mesoporous materials is TEOS, so the nanomaterial synthesis is used TEOS ${ }^{7,10-11}$.
Besides TEOS, CTAB is also one of the components of mesoporous Gd-silica. CTAB acts as a surfactant has hydrophilic groups that can bind water and have hydrophobic groups that cannot bind water, CTAB can unite gadolinium in a watersoluble acidic atmosphere with insoluble silica in water. CTAB is one of the cationic type surfactants that have a positive charge, because according to ${ }^{14}$ on polymerization process there will be inhibition in the presence of nonionic and anionic surfactants.

When all the constituent components have been mixed, the atmosphere in the solution is acidic, but the synthesis of mesoporous Gd-silica is optimum at $\mathrm{pH}$ 9. Therefore, ammonium hydroxide was added to the optimum condition that $\mathrm{pH} 9$ was reached on the system. Due to the acidic atmosphere, gadolinium will bind to chloride from hydrochloric acid, thereby increasing the likelihood of formation of toxic gadolinium (III). The fine particles of formed silicon gadolinium are heated at $80^{\circ} \mathrm{C}$ with sufficiently strong and constant stirring to remove the ethanol formed from the hydrolysis and condensation processes of $\mathrm{SiO}_{2}$ formation and the formation of the gadolinium sol. After ethanol was released from the system, centrifugation is done to separate the solvent with the gel formed because it was already separated from the solvent. The centrifugation aims to purify the silica gadolinium gel from solvents and other impurities by rotating by centrifugal force to separate between the supernatant and its sediment ${ }^{12}$.

After forming gadolinium silica gel, the gel was calcinated at $600^{\circ} \mathrm{C}$ for 6 hours. Calcination aims to convert a particular compound to its oxidant compound without the addition of reagents, but there will be decomposition. This calcination produces white mesoporous $\mathrm{Gd}$-silica solids. The results of this synthesis resulted in an average yield of $97.93 \%$. In addition to Gd-MS, synthesized mesoporous silica act as a blank for comparison with Gd-MS.

\section{Characterization of Mesoporous Silica Gadolinium Powder by using TEM}

Gadolinium mesoporous silica was analyzed using TEM to determine the morphology of compounds and pore size. From the results of TEM analysis, it is found that the morphological form approaches the sphere but is less uniform overall. In addition to the morphology, the TEM obtained a representative pore size of gadolinium mesoporous 
silica samples of about $30-50 \mathrm{~nm}$ by expected results because according to mesoporous a pore that has sizes in the range of 2-50 nm. Fig. 1 illustrates the morphology of the TEM analysis and its particle size in Table 1.

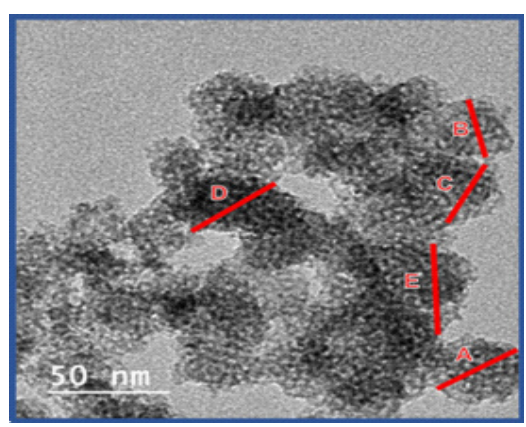

Fig. 1.TEM Gd-MS analysis

In Table 1 the pore size of the gadolinium mesoporous silica synthesized is in the range $<50 \mathrm{~nm}$. From this pore size, the average pore size is $38.184 \mathrm{~nm}$ with a standard deviation of 4.76 . In addition to the pore size in Table 1, also obtained the surface value of gadolinium mesoporous silica which is high at $580.666 \mathrm{~nm}$.

Table 1: Gd-MS pore size

\begin{tabular}{cc}
\hline & Pore Size $(\mathrm{nm})$ \\
\hline A & 35.653 \\
B & 32.430 \\
C & 40.849 \\
D & 37.231 \\
E & 44.756 \\
X & 38.184 \\
\hline
\end{tabular}

Analysis of Gd (III) with Spectrophotometer of Visible Light

Gadolinium in the form of $\mathrm{Gd}^{3+}$ ions was analyzed by using visible light spectrophotometers to find out the $\mathrm{Gd}^{3+}$ concentration of synthetic gadolinium mesoporous silica. Determination of this concentration using acetic acid buffer as solvent of xylenol orange (XO) which become indicator of existence of $\mathrm{Gd}$ (III). Xylenol orange used a $\mathrm{pH} 5.8$ acetate buffer solution because $\mathrm{XO}$ was stable at that $\mathrm{pH}$ and the metal would bind to $\mathrm{XO}$ under $\mathrm{pH}$ 5.8. The metal that will bind to this $\mathrm{XO}$ is gadolinium which will be determined its concentration because this Gd (III) will be toxic to the human body when it is shaped as its ion. Therefore, determined the concentration of Gd (III) to be known gadolinium mesoporous silica synthesized will result in toxic effects or not.
XO measured its maximum wavelength first by using a visible light spectrophotometer at a wavelength range of $400-700 \mathrm{~nm}$. From the results of determining the maximum wavelength, it was found that $\mathrm{XO}$ absorbs the maximum wavelength at $570 \mathrm{~nm}$, so that in the determination of absorbance of the standard solution and gadolinium mesoporous silica sample was determined at a wavelength of $570 \mathrm{~nm}$. At the time of making the standard curve of gadolinium made variation of concentration 2, 3, 4, and 5 ppm generated curve as in Fig. 2. From standard curve result, gadolinium mesoporous silica sample concentration measured $0.178 \mathrm{ppm}$ with limit of gadolinium (III) allowed in body only $50 \mathrm{ppb}$. This value of $0.178 \mathrm{ppm}$ if converted to milligram would be $3.56 \times 10^{-3} \mathrm{mg}$.

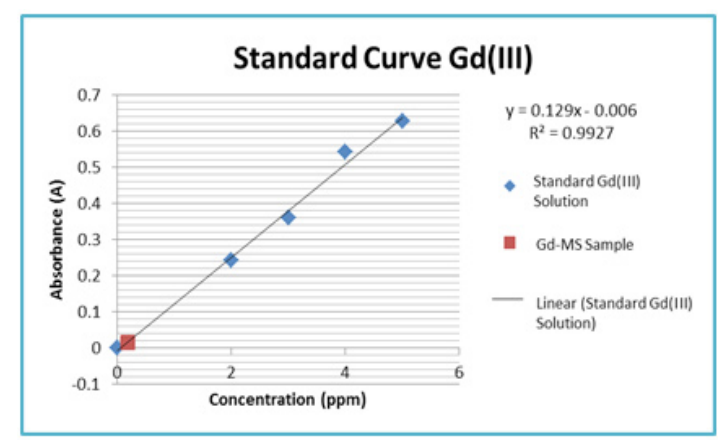

Fig. 2. Standard curve of gadolinium(III)

Measured concentration is due to the presence of gadolinium (III) which is pretty much bound with $\mathrm{XO}$. When gadolinium (III) is not tied to $\mathrm{XO}$, gadolinium dissolves in a colorless solution, while when gadolinium (III) is bound to XO the color of the solution will turn purple. The XO has an orange color when it does not bind to a metal.

\section{Stability Test of Gadolinium Mesoporous Silica}

Gadolinium mesoporous silica was tested at a temperature of $37.5^{\circ} \mathrm{C}$ because it was a temperature that resembled human body temperature. Gadolinium mesoporous silica is expected in the future can be done clinical trials, then for the preliminary tests conducted at the same temperature conditions. Samples of gadolinium mesoporous silica were incubated at $37.5^{\circ} \mathrm{C}$ for 24 $\mathrm{h}$, because the clinical trials of the contrast agent in the body were only 24 hours. After $24 \mathrm{~h}$, gadolinium mesoporous silica samples will pass through the kidney then into the anus. After incubation at $37.5^{\circ} \mathrm{C}$ for $24 \mathrm{~h}$, gadolinium mesoporous silica samples were titrated to determine the concentration 
of gadolinium released from the silica trap as a gadolinium (III).

After the titration to determine the concentration of gadolinium (III), obtained gadolinium (III) regardless of mesoporous silica with the concentration value of the Gd-MS sample is 4.020 $\times 10^{-3} \mathrm{~N}$. From that concentration it can be converted to a milligram value of $6.32 \times 10^{-2} \mathrm{mg}$. Therefore, it can be stated that gadolinium is still present which is detached from the synthesized structure of mesoporous silica structure. There was a change of color from red wine to blue titration result of $\mathrm{Na}$-EDTA standardization by $\mathrm{MgSO}_{4} \cdot 7 \mathrm{H}_{2} \mathrm{O}$ by using EBT indicator. The result of this standardization resulted in Na-EDTA concentration of $0.0201 \mathrm{~N}$.

Titration of stability test results using $\mathrm{XO}$ indicator obtained the color change from purple to yellow. From the stability test results, gadolinium mesoporous silica has a good stability at $37.5^{\circ} \mathrm{C}$ for 24 $\mathrm{h}$, but there are still $6.32 \times 10^{-2} \mathrm{mg}$ of gadolinium lose from the silica skeleton so it must be prepared further to be used as an agent contrast to the patient.

\section{CONCLUSION}

Gadolinium and silica in the mesoporous form interact physically and have an average pore size of $38.184 \mathrm{~nm}$. All these results demonstrate that the Gd-MS product is more suitable to be used as an alternative for synthesizing gadolinium-based MRI contrast agents.

\section{ACKNOWLEDGMENT}

The authors would like to thank the various parties who have helped in this research, especially to Academic Leadership Grant (ALG) Universitas Padjadjaran Bandung Indonesia.

\section{REFERENCES}

1. Fauzia, R. P.; Mutalib, A.; Sodjanaatmadja, R.U.M. S.; Anggraeni, A.; Bahti, H.H. Chimica et Natura Acta., 2016, 4, 7-15.

2. Ratzinger, G.; Agrawal, P.; Körner, W.; Lonkai, J.; Sanders, H. M. H. F. E.; Terreno, M.; Wirth, G. J.; Strijkers, K.; Nicola, F.; Gabor, F. Biomaterials., 2010, 31, 8716-8723.

3. Feldmann, V.; Engelmann, J.; Gottschalk, S.; Mayer, H. A.; J. Colloid and Interface Science., 2012, 366, 70-79.

4. Gupta, N.; Shrivastava, A.; Sharma K. R. Int. J. Nanomedicine., 2012, 7, 5491-5500.

5. Nandiyanto, A.B.D.; Kim, S.G.; Iskandar, F.; Okuyama, K. Microporous and Mesoporous Materials., 2009, 120, 447-453.

6. Bertini, I.; Luchinat, C.; Parigi, G.; Ravera, E. NMR of Paramagnetic Molecules (Second Edition)., 2015.

7. Lin, C.F.; Zhang, X.; Lin, H.; Wang, N.; Li, J. B.; Yang, X. Z. Advanced Materials Research., 2006, 11-12, 543-546.

8. Lin, Y.S.; Hung, Y.; Su, J. K.; Lee, R.; Chang,
C.; Lin, M. L.; Mou, C.Y. J. Physical Chemistry B., 2004, 108, 15608-15611.

9. Barge, A.; Cravotto, G.; Gianolio, E.; Fedeli, F. Contrast Med. Mol. Imaging., 2006, 1, 184-188.

10. Eddy, D.R.; Dwiyanti, D.; Rahayu, I.; Hastiawan, I.; Bahti, H.H. AIP Conference Proceedings., 2017, 1848, 030003-1.

11. Rahayu, I.; Indraneli, R. P.; Yuliyati, Y.B.; Anggraeni, A.; Soedjanaatmadja, R. U. M. S.; Bahti, H.H. J. Physics, Conference Series ., 2018, 1013, 012197-1.

12. Fauzia, R.P.; Mutalib, A.; Soedjanaatmadja, R.U.M.S; Bahti, H.H.; Anggraeni, A.; Gunawan, A.H.; Hidayati, Y. Procedia Chemistry., 2015, 17, 139-146.

13. Eddy, D.R.; Rahayu, I.; Wyantuti, S.; Hartati, Y. W.; Firdaus, M. L.; Bahti, H. H. J. Physics: Conference Series., 2018, 1080, 012013.

14. Zhang, T.; Blum, F. D. J. Colloid Interface Science., 2017, 504, 111-114. 